Chirurg 2015 $\cdot 86: 802$

DOI 10.1007/s00104-015-0052-6

Online publiziert: 1. Juli 2015

(c) Springer-Verlag Berlin Heidelberg 2015

CrossMark

J. Reibetanz · C.T. Germer

Klinik für Allgemein-, Viszeral-, Gefäß- und Kinderchirurgie, Universitätsklinik Würzburg, Würzburg, Deutschland

\title{
Laparoskopische vs. offene Rektumkarzinomchirurgie
}

\section{3-Jahres-Ergebnisse der COLOR-II-Studie}

Schnittbildgebung (Computertomographie oder Magnetresonanztomographie) des Beckens durchgeführt, zusammen mit einer Bildgebung von Leber und Lunge.

\section{Ergebnisse}

\section{Einleitung und Fragestellung}

Die bereits 2013 publizierten Kurzzeitergebnisse der Studie „Colorectal Cancer Laparoscopic or Open Resection“ (COLOR-II) zeigten, dass die offene und laparoskopische TME („total mesorectal excision“) hinsichtlich ihrer Morbiditäts-/ Komplikationsraten vergleichbare Resultate liefern, bei leichten Vorteilen im perioperativen Outcome für den minimalinvasiven Zugang. Darüber hinaus blieb durch die Laparoskopie die onkologische Radikalität des Eingriffs gewahrt [1]. Aktuell werden die 3-Jahres-Lokalrezidivund Überlebensraten der COLOR-II-Studie vorgestellt.

\section{Methode}

Die COLOR-II-Studie ist eine multizentrische, 2:1 randomisierte Phase-III-Studie zum Vergleich der laparoskopischen mit der offenen TME beim nichtmetastasierten, CRM („circumferential resection margin“) -negativen Rektumkarzinom $\leq 15 \mathrm{~cm}$ ab ano. Primärer Endpunkt von COLOR-II ist die 3-Jahres-Lokalrezidivrate. Sekundäre Endpunkte beinhalten das krankheitsfreie und Gesamtüberleben. Das Follow-up verlangte mindestens eine jährliche klinische Untersuchung bis zum 5. postoperativen Jahr. Zudem wurden 3 Jahren nach dem Primäreingriff eine
Im Zeitraum zwischen Januar 2004 und Mai 2010 konnten für COLOR-II insgesamt 1044 Patienten ausgewertet werden, von denen 699 (67\%) laparoskopisch und 345 (33\%) offen operiert worden waren. Für das Langzeit-Follow-up standen hinsichtlich der Endpunkte „Lokalrezidivrate“, „krankheitsfreies Überleben“ bzw. „Gesamtüberleben“ die Daten von 771 (74\%), 923 (89\%) bzw. 903 (87\%) Patienten zur Verfügung. Nach 3 Jahren postoperativ lag die Lokalrezidivrate für offen und laparoskopisch operierte Patienten jeweils bei 5,0\%. In der Subgruppe der tiefen Rektumkarzinome unterschied sich die Lokalrezidivrate in der „As-treatedPopulation“ zwischen beiden Therapiearmen jedoch deutlich zugunsten der laparoskopischen Rektumresektion (3,5 vs. 12,7\%). Das krankheitsfreie Überleben war nach 3 Jahren für laparoskopisch bzw. offen operierte Patienten mit 74,8 bzw. $70,8 \%$ vergleichbar, ebenso das 3-JahresGesamtüberleben mit 86,7 vs. $83,6 \%$. In der Subgruppe der UICC-III-Patienten zeigte sich jedoch hinsichtlich des krankheitsfreien Überlebens ein Vorteil zugunsten der Laparoskopie (52,0 vs. 64,9\%).

\section{Diskussion und Fazit des Reviewers}

Höchstrangig publiziert werden aktuell die Langzeitergebnisse der COLOR-II-
Studie vorgestellt. Hier bestätigt sich, dass die laparoskopische Rektumkarzinomchirurgie der offenen Resektion hinsichtlich des primären Endpunkts „Lokalrezidivrate", aber auch in Bezug auf krankheitsfreies und Gesamtüberleben (mindestens) gleichwertig ist. Beim tiefen Rektumkarzinom ist die Laparoskopie der offenen TME - wahrscheinlich aufgrund der besseren Visualisierung im kleinen Becken - hinsichtlich CRM-Positivität (Risikoreduktion: $-11 \%$ ) sowie Lokalrezidivrate (Risikoreduktion: $-8,9 \%$ ) sogar überlegen. Betrachtet man jedoch die bereits 2013 publizierten Kurzzeitergebnisse von COLOR-II wurde dieser onkologische Benefit beim tiefen Rektumkarzinom mit einer vergleichsweise hohen Rate an Kontinenzverlust erkauft ( $53 \%$ in der Gruppe der laparoskopischen Resektionen vs. $23,5 \%$ in der Gruppe der offenen Resektionen; [1]).

\section{Korrespondenzadresse}

\section{J. Reibetanz}

Klinik für Allgemein-, Viszeral-, Gefäßund Kinderchirurgie Universitätsklinik Würzburg, 97080 Würzburg reibetanz_j@ukw.de

\section{Einhaltung ethischer Richtlinien}

Interessenkonflikt. J. Reibetanz und C.T. Germer gibt an, dass kein Interessenkonflikt besteht.

\section{Literatur}

1. Pas MH van der, Haglind E, Cuesta MA et al (2013) Laparoscopic versus open surgery for rectal cancer (COLOR II): short-term outcomes of a randomised, phase 3 trial. Lancet Oncol 14:210-218 